\title{
Assessment of non-technical skills of operating room nurses
}

\author{
Anna Ribakova and Liana Deklava \\ Rīga Stradinsš University, Latvia
}

\begin{abstract}
Training of non-technical skills helps to achieve reduction of human errors that could contribute to safety of patients. For assessment of non-technical skills of Operating Room (OR) nurses, researchers of the University of Aberdeen developed intra-operative work organization protocol for observation of non-technical behaviour. This system includes taxonomy of non-technical skills, definitions, desirable and adverse behavioural markers, and Likert scale for behavioural assessment. The objective of this research is to assess non-technical skills of OR nurses in work environment and compare the findings with OR nurses self-assessment of non-technical skills. The study involved 15 interviews with OR nurses in sterile position (scrub nurses) and 15 observations of their work in four hospitals. Providing self-assessment, OR nurses note a tendency to minimal communication. In practice, nurses often show good ability to think analytically, to predict events and needs and are able to act decisively during surgery. OR nurses in Latvia partially associate their work with nontechnical skills, however in practice these skills are used, and they were relatively highly valued during the study. Insufficient self-assessment of such skills of nurses as cooperation in performance of physical tasks, promotion of personnel safety and decisive action, indicates the need for development of scrub nurse's work standards with clearly defined area of responsibility and duties.
\end{abstract}

Key words: non-technical skills, operating room nurse, scrub nurse, OR nurse's skills.

\section{Introduction}

One can easily notice an assured, efficient and skilled OR nurse during an operation. Some nurses are able to keep the situation in control even if the surgical procedure is not going as initially planned. Some of them are able to stay calm and solve problems depending on the situation while providing qualified help for the surgical team. In addition to technical skills and knowledge, these OR nurses demonstrate good "non-technical skills" that complement the technical skills to carry out tasks in a safe and efficient manner.

Non-technical skills refer to general or life skills that are not part of formal education programs. These skills are not meant for any specific work conditions or work environment but they can be widely used in any work environment and with any tasks. It is not possible to learn these skills in a short period of time, because it requires a wide application range and significant experience. Non-technical skills contribute to the skills, necessary for certain tasks in the work environment. In addition, non-technical skills have an important role in the maintaining of the technical skills [1]. 
Non-technical skills are part of the competences required for a OR nurse. A competence is a human characteristic that is in a causal relationship with effective and outstanding work performance and is based on certain criteria [2].

Non-technical skills differ from the psychomotor skills because they include cooperation between team members (e.g. communication, teamwork and leadership) or thinking skills, for example, the ability to recognize and understand a situation or make decisions. When combined, it helps to carry out tasks [3]. A few categories of non-technical skills, for example, task management include both cognitive and social elements.

It is hard to measure non-technical skills because it is a broad concept that includes several aspects that can be essential depending on the situation. Furthermore, the majority of non-technical skills are automatic and consist of routine behaviour, and often people do not realize to what extent they are using these skills. The best way of assessing the nontechnical skills of the personnel is the observation using a specific method depending on the field [4].

Due to the fact that non-technical skills are integrated in technical skills, the same method of assessment can be used for both types. During observation, special protocols and simulation methods developed by researchers are used because, when using actors who play the roles of the patients, it is a simple way of developing the skills. Mannequins and high-precision mannequins-stimulators are also widely used. Skills developed in an artificial environment can then be easily transferred to real-life situations [5].

Self-assessment is often used as a method of assessment of competences. According to research, this method has its advantages: it is cost effective; it helps nurses to maintain and improve their work by determining the strengths and areas where improvement is possible; it helps nurses to control their work $[6,7]$.

\section{Objective}

To assess non-technical skills of OR nurses in work environment and compare the findings with OR nurses self-assessment of non-technical skills.

\section{Sampling}

The study sample consists of 15 OR nurses with an average work experience of 20.6 years from four hospitals in Riga and its region. Respondents were chosen by the following criteria: experience in scrub nurse position in an operation room no less than five years, profile of observed operations - abdominal surgery.

\section{Methods}

To reach the objective qualitative research methods were used: 15 observations of the work of OR scrub nurses and 15 interviews. The study instruments: "Scrub Practitioners' List of Intra-operative Non-Technical Skills" (SPLINTS), the observation instrument developed by the Aberdeen University, that was translated and adapted into Latvian. The questions for the interview were developed based on the SPLINTS observation protocol and they were identical for both groups of respondents.

The electronic version of the instrument was acquired after reaching an agreement with the representative of the Aberdeen University. The instrument was developed in 2010 by a specialist multidisciplinary team from Scotland.

It is a field-specific behaviour assessment system which is used to identify, observe and develop the non-technical skills of OR nurses in sterile position. 
Table 1. Assessment of the non-technical skills of the OR nurses sample by the means of observation protocol SPLINTS.

\begin{tabular}{|l|c|c|c|c|c|}
\hline $\begin{array}{l}\text { Non-technical skills } \\
\text { Category/element }\end{array}$ & $\begin{array}{c}\text { Number of } \\
\text { cases, } N\end{array}$ & $\begin{array}{c}\text { The minimum } \\
\text { value }\end{array}$ & $\begin{array}{c}\text { The maximum } \\
\text { value }\end{array}$ & $\begin{array}{c}\text { The mean } \\
\text { value }\end{array}$ & $\begin{array}{c}\text { Stat. } \\
\text { deviation }\end{array}$ \\
\hline Situation awareness & 15 & 3 & 4 & 3.73 & 0.46 \\
\hline Acquisition of information & 15 & 3 & 4 & 3.73 & 0.46 \\
\hline $\begin{array}{l}\text { Recognition of information } \\
\text { and understanding }\end{array}$ & 15 & 2 & 4 & 3.53 & 0.74 \\
\hline Prediction & 15 & 3 & 4 & 3.67 & 0.49 \\
\hline Communication and teamwork & 15 & 3 & 4 & 3.80 & 0.41 \\
\hline Decisive action & 15 & 3 & 4 & 3.87 & 0.35 \\
\hline Information exchange & 15 & 2 & 4 & 3.47 & 0.74 \\
\hline Coordination with other persons & 15 & 3 & 4 & 3.80 & 0.41 \\
\hline Task management & 15 & 3 & 4 & 3.67 & 0.49 \\
\hline Planning and preparation & 15 & 3 & 4 & 3.60 & 0.51 \\
\hline $\begin{array}{l}\text { Regulation and maintenance } \\
\text { of standards }\end{array}$ & 15 & 2 & 4 & 3.33 & 0.62 \\
\hline Stress management & 15 & 3 & 4 & 3.87 & 0.35 \\
\hline
\end{tabular}

The instrument contains:

- The definitions of non-technical skill categories and their elements; the taxonomy used in the document is a three-level hierarchy with three highest level categories and nine elements that apply to these qualification categories;

- Good and bad behaviour examples that are used as markers during the observation of behaviour;

- A rating scale that is used to measure the observed behaviour and acquiring feedback. It is based on the Likert scale with values from 1 (poor), 2 (nearly poor), 3 (acceptable), 4 (good) [8].

Before the assessment of the non-technical skills of the OR nurses, the tool was translated, adapted and tested in accordance with the International Test Commission Guidelines for test translation and adaption (International Test Commission, 2010). This process involved the translation of the instrument by respecting the context of the cultural environment, testing of the translated instrument, documentation of the administration and adaption of the instrument and designing the guidelines for interpreting the assessment included in the instrument.

\section{Results}

During the observation phase of the study, the non-technical skills of 15 OR nurses were assessed by the means of the protocol of "Scrub Practitioners' List of Intra-operative NonTechnical Skills" (SPLINTS). Study participants were told that the objective of the observer is to observe the course of the surgery and to use the resulting data for research, however, the nature of the data was not emphasized.

OR nurses generally show rather high non-technical skills. The highest mean ratio of 3.87 $(\mathrm{SD}=0.35)$ was observed in the category "Stress Management", the lowest mean ratio of 3.33 ( $\mathrm{SD}=0.62$ ) was observed in the category of "Regulation and maintenance of standards" which can be associated with non-compliance with the personnel safety requirements. The 
lowest ratio of 2 (nearly poor) was observed in categories "Recognition of information and understanding", "Information exchange" and "Regulation and maintenance of standards".

\subsection{Situation awareness}

The descriptive statistical data collected during the observations shows that OR nurses are actively using the acquisition of information skills during work. The observing and listening skill is used fully $(n=15)$ and during self-assessment all nurses $(n=15)$ confirmed using this particular skill. The collected observation data indicates that nurses are also using questions to acquire information $(n=11)$, however, in self-assessment only $1 / 3$ of the OR nurses confirm using this skill.

To acquire information it is also important to recognize the signals of the operation room environment and during observation it was noted that all nurses $(n=15)$ demonstrated this skill, and in the self-assessment almost every nurse $(n=14)$ confirmed that this skill was used. All nurses $(n=15)$ pay attention to the groups of operation room signals: surgical process, patient and team members; attention to devices was paid by 14 nurses. The selfassessment data displayed a different picture and it can be noted that majority of the respondents recognize the signals of surgical process and team members, but signals of the patient or devices are recognized rarely - only $1 / 5$ of the nurses emphasized the importance of these particular signals.

The skill "Recognition of information and understanding" anticipates that nurses will be able to recognize and understand the acquired information and data collected during the observation confirm that nurses use this skill to its full extent. In the self-assessment the results are only slightly lower $(n=13)$. Another key skill of OR scrub nurse is to compare the acquired information with the current knowledge, for example, pass the right medical equipment before the surgeon asks for it or the wrong piece of equipment is requested as well as ability to understand a specific situation. During the observation phase all nurses displayed this skill $(n=15)$ but self-assessment data values were lower $(n=10)$. Understanding of a specific situation was noticed in the work of all nurses, but only $2 / 3$ confirmed using this skill during the self-assessment.

The prediction skill was shown by all nurses $(n=15)$ and all but one $(n=14)$ nurse emphasized the importance of this skill in the self-assessment. The ability to predict events received the lowest values in the prediction category -12 nurses used it in practice and during self-assessment it was confirmed by 11 of them. All of the nurses $(n=15)$ displayed their analytical thinking and prediction skills to predict what might be necessary as the surgery progresses. In the self-assessment, half of the nurses confirm using analytical thinking.

\subsection{Communication and teamwork}

The data collected during the observation shows that all the nurses are able to act decisively during an operation and in the self-assessment the results are similarly high - 13 nurses confirmed using this particular skill. In practice the majority of nurses $(n=13)$ ask for clarifications and explanations and during self-assessment nurses often $(n=11)$ rated this skill positively. The observation data also indicates that all 15 nurses adjust their style of communication depending on the situation but during self-assessment more than half $(n=9)$ of the nurses affirmed adjusting their communication.

It was observed that 14 of the nurses act with a fitting level of assurance, which means that a nurse can take on the role of a team leader, but in the same time also knows the limit of her possibilities. More than $2 / 3$ of the respondents talk about this skill in the self-assessment. Often decisive actions are linked to carrying out a report during surgery and this opinion is 
Table 2. Descriptive statistical data of the category "Situation awareness".

\begin{tabular}{|c|c|c|c|}
\hline Element & Components of the element & Observation, $\mathrm{N}$ & $\begin{array}{c}\text { OR nurse } \\
\text { self-assessment, } \mathrm{N}\end{array}$ \\
\hline \multirow{3}{*}{$\begin{array}{l}\text { Acquisition } \\
\text { of information }\end{array}$} & Observing and listening & 15 & 15 \\
\hline & Asking questions & 11 & 5 \\
\hline & $\begin{array}{l}\text { Recognizing signals of the } \\
\text { operation room environment }\end{array}$ & 15 & 14 \\
\hline \multirow{4}{*}{$\begin{array}{l}\text { Sources of the operation } \\
\text { room environment signals }\end{array}$} & Surgical process & 15 & 11 \\
\hline & Devices & 14 & 3 \\
\hline & Patient & 15 & 3 \\
\hline & Surgical team members & 15 & 14 \\
\hline \multirow{3}{*}{$\begin{array}{l}\text { Recognition of information } \\
\text { and understanding }\end{array}$} & $\begin{array}{l}\text { Recognition and interpretation } \\
\text { of information }\end{array}$ & 15 & 13 \\
\hline & $\begin{array}{c}\text { Comparison of information } \\
\text { with knowledge }\end{array}$ & 15 & 10 \\
\hline & $\begin{array}{l}\text { Understanding of specific } \\
\text { situations }\end{array}$ & 15 & 10 \\
\hline \multirow{4}{*}{ Prediction } & Prediction skills & 15 & 14 \\
\hline & Analytical thinking skills & 15 & 7 \\
\hline & Ability to predict events & 12 & 11 \\
\hline & Ability to predict needs & 15 & 11 \\
\hline
\end{tabular}

Table 3. Descriptivestatistical data of the category "Communication and teamwork".

\begin{tabular}{cccc}
\hline \multirow{2}{*}{ Element } & Components of the element & Observation, N & $\begin{array}{c}\text { OR nurse } \\
\text { self-assessment, N }\end{array}$ \\
\hline \multirow{2}{*}{ Decisive actions } & $\begin{array}{c}\text { Can act decisively } \\
\text { Asks for clarifications } \\
\text { and explanations }\end{array}$ & 13 & 13 \\
& $\begin{array}{c}\text { Adjusts communication } \\
\text { depending on the situation }\end{array}$ & 15 & 11 \\
& Uses fitting level of assurance & 14 & 9 \\
\hline \multirow{2}{*}{ Information exchange } & $\begin{array}{l}\text { Asks for information } \\
\text { Provides information }\end{array}$ & 12 & 11 \\
\hline \multirow{2}{*}{ Coordination with others } & Exchanges opinions and ideas & 15 & 5 \\
& $\begin{array}{c}\text { Cooperation during physical tasks } \\
\text { Actions to be taken contribute } \\
\text { to the surgery procedure }\end{array}$ & 15 & 7 \\
& & 15 & 10 \\
\hline
\end{tabular}

supported by 6 respondents, 5 nurses link decisive actions to providing a sterile environment and 2 respondents talk about aspects of leading the surgical team.

Information exchange has a very important role in the work of a surgical team. Observation data indicates that 12 OR nurses asked for information during the operation, however, in the self-assessment such results were not achieved - almost $2 / 3$ of the nurses said they asked for information. Regarding providing information during an operation, it was noted that it was done by 14 nurses, but in the self-assessment only $1 / 3$ of the study sample rated this skill positively.

After collecting data during observation it was concluded that during work nurses are also using non-verbal communication. A combination of verbal and non-verbal communication was used by 8 nurses. 
Table 4. Descriptive statistical data of the category "Task management".

\begin{tabular}{cccc}
\hline Element & Components of the element & Observation, N & $\begin{array}{c}\text { OR nurse } \\
\text { self-assessment, N }\end{array}$ \\
\hline Planning and & Time planning & 15 & 11 \\
preparation & Organizing needs & 15 & 15 \\
& Organizing actions & 15 & 13 \\
\hline Regulation and maintenance & Personnel safety & 14 & 3 \\
of standards & Patient safety & 15 & 11 \\
& Good practice guidelines & 15 & 9 \\
\hline \multirow{2}{*}{ Stress management } & Overcomes stress situations & 1 & 7 \\
& Keeps calm & 15 & 11 \\
& Understands needs of others & 14 & 8 \\
& Understands stress of others & 8 & 9 \\
\hline
\end{tabular}

Observation data reveal that when coordinating with others all 15 nurses are sharing their opinion and ideas, for example, if any deviations from the initial plan are going to happen or if the plan needs to be clarified but only 7 nurses mentioned exchanging opinions and ideas in the self-assessment. It was also noted that all of the nurses were able to cooperate with the surgeons when carrying out physical tasks, for example, isolating the surgery area with a sterile cloth or connecting medical devices. In the self-assessment this skill was rated positively by only $2 / 3$ of the nurses $(n=10)$.

To contribute to a successful surgery procedure it can be important to prioritize simultaneous requests from different members of the surgical team. During observation, all 15 nurses demonstrated such skill but in the self-assessment it was confirmed by 11 nurses.

\subsection{Task management}

During self-assessment on the skills of task management, 14 OR nurses said that they are associating it with planning and organizing the work. Two of nurses associated it with maintaining different standards and just one nurse associated it with lowering the stress levels of the whole team.

The collected data shows that in practice the OR nurses show excellent skills during planning and preparation. All of the nurses $(n=15)$ displayed that they are able to plan the time and the majority of nurses $(n=11)$ in the self-assessment note that they prefer to manage time in a way that ensures that others do not have to wait. Another task of a OR nurse is to organize the needs of the surgical team (equipment, devices etc.) that are connected with the surgical procedure. During self-assessment this skilled was valued positively by all nurses $(n=15)$. It is necessary for a nurse to organize her own actions, and in practice, all of the nurses were able to do it. During self-assessment this skill was mentioned by 13 nurses.

During observation, the personnel safety from the regulation and maintenance of standards category was noted in 14 cases. This skill was not used in one case, and was used partly regarding personnel not wearing safety eyewear. In self-assessment, only 3 nurses pay attention to the safety of personnel.

The requirements of the patient safety are fulfilled in practice by all 15 nurses, in selfassessment nurses report applying patient safety measures that are connected to ensuring a sterile surgical environment and keeping the record during the operation in 11 cases.

The good practice guidelines are met by all of the nurses but in self-assessment more than a half $(n=9)$ of the nurses mention this skill. 
Two of the nurses pointed out that there are no common standards regarding OR nurses and that it would be necessary to draw up standards to ensure the quality of their work.

OR nurses displayed good stress management skills. All of them were able to stay calm. The majority $(n=14)$ of the nurses are able to understand the needs of surgical team members as well as control and organize the technical measures accordingly. During the observation, one of the nurses overcame a stress situation but 8 nurses showed their ability to understand stress of the surgical team members.

During the interview, almost half of the nurses mentioned situations when it was necessary to overcome stress during and operation. 2/3 of the nurses gave a positive selfassessment about their abilities to stay calm but more than a half said they were able to understand the needs as well as the stress of other surgical team members and carry out actions that lower the stress of the whole team.

\section{Discussion}

After evaluating the non-technical skills of OR nurses in scrub position, results show comparatively high values with an average grade of $3.68(\mathrm{SD}=0.14)$ over 15 observations. Such results could be explained by that fact that the study sample was OR nurses with an average work experience as scrub nurse of $20.6(\mathrm{SD}=12.01)$ years.

\subsection{Situation awareness}

There are certain sources of information referring to non-technical skills of OR nurses during surgery: surgical process, medical devices, surgical team and the patient. When comparing the study data it can be concluded that there are some differences regarding the understanding of the operation room signals. In practice, nurses demonstrate good skills of recognizing the signals of medical devices and patients, however, in the self-assessment only 3 nurses confirm using this particular skill. This data may indicate that the importance of signals given by the medical devices and patient is not high enough which could be the cause of insufficient safety of the patient during an operation.

The recognition and interpretation of information expects OR nurses to be able to recognize information, to compare it to their existing knowledge and to understand a specific situation, for example, when sudden changes occur during the operation. In practice this skill is used by all nurses, however in the self-assessment the results are lower.

Prediction skills include analytical thinking, prediction of the events and prediction of the possible needs in the near future (medical equipment, suture materials, medical devices etc.). Comparison of the self-assessment data and the observation data shows that there is no large difference in the results of prediction skill usage. During the self-assessment, every third nurse noted that prediction skills improve with experience.

\subsection{Communication and teamwork}

In the interview, nurses self-assessed the communication used when working in a team. The majority of the OR nurses are exchanging information with other members of the surgical team. Less than a half of the nurses admit using a style of communication that leads to a successful operation. More than a half of the nurses believe that minimal communication is the most fitting style of communication during an operation. This data makes one consider if the style of communication used currently provides a sufficient information exchange between the members of the surgical team to accomplish a single goal? Does it contribute to the safety of the patients? In opinion of many researchers insufficient communication is 
the main reason for any mistakes made during an operation. It is noted by Makary and his colleagues [9], and Mill [10].

OR nurses must be able to act decisively in the limits of their competence. The comparison of data collected during interviews and data from observations leads to significant differences. Respondents relate decisive actions to keeping records during the operation and providing a sterile surgical environment. The relation of these actions to the coordination of the work of the surgical team is not noted very often. Respondents did not mention that decisive actions could be linked to taking care of the patient or a nurse taking the role of the team leader if that is in her competence. These results coincide with the work of Mitchell and Flin, who emphasize that leading of a surgical team is not within the competence of a OR nurse, and the significance of this skill may be shown in cases when nurse is working together with a surgeon or inexperienced medical personnel, or when maintaining high standards of medical care (Mitchell and Flin, 2008).

In his work, Pike names the inner factors that limit the leadership competence of a nurse and they are: uncertainty, insufficient assurance, lack of professional trust, shyness, fear, insecurity, inferiority [11].

Observation indicates that the majority of the nurses are able to act decisively during an operation; however, in the self-assessment this skill has slightly lower values.

Comparing the data on the use "Information exchange" skills, it can be concluded that the expectations of surgeons and self-assessment of nurses are low, but data collected during the observation indicates that nurses are actually using these skills in practice but the selfassessment of these skills was rather average.

\subsection{Task management}

Interviewees usually connect task management with the planning and organization of work and rarely with the maintenance of standards. In the self-assessment, one nurse mentioned lowering the stress of the team as a management task.

In the observation, all of the nurses displayed their planning and organizing skills to the fullest. In the self-assessment, a slightly lower number or nurses mention that they are organizing their own actions and planning time to ensure that others do not have to wait.

Self-assessment regarding personnel safety is very low. The observation data shows that personnel safety requirements are met partly, and most frequently, this skill is not active by not using suitable safety eyewear. These results coincide with the study about the use of strategies and methods of lowering the risks of personnel infections and injuries during operations that was carried out in 2010. It was found out that only 29 per cent of the respondents wear safety eyewear during operations.

The requirements of the guidelines on patient safety and good practice were fulfilled by all nurses. OR nurses are not only able to tolerate stress during their work and take actions that lower the stress of the surgical team members but also understand their stress and take up the role of a comforter.

\section{Conclusions}

In the result of the study the list of scrub practitioners' intra-operative non-technical skills or an observation protocol was translated, adapted and tested in practice. An assessment of the OR nurse in sterile position non-technical skills was also carried out.

The information exchange between the OR nurses and the surgical team if not sufficient because in the OR nurse self-assessment there is a tendency to more often provide information rather than ask for it. 
Data about the fact that during a surgery OR nurses pay more attention to the signals of the surgical process or the surgical team and do not provide information about the signals given by the medical equipment or the patient may indicate the lack of attention to the safety of the patient.

The results about the cooperation of OR nurses when carrying out physical tasks, the safety of the personnel and decisive actions of OR nurses indicates that nurse knowledge about their competences is not sufficient and once again it emphasizes the need to develop common standards that would precisely define the responsibilities and competences of OR nurses.

In practice OR nurses are able to tolerate stress during surgery, take actions that lower the stress levels of the surgical team and provide emotional support if necessary.

The study indicates that non-technical skills are not a well-known concept between the study sample.

\section{References}

[1] A. Nasir, D.F. Ali, M. Noordin, M.S. Nordin, Technical skills and non-technical skills: predefinition concept Proceedings of the IETEC'11 Conference, Kuala Lumpur, Malaysia

[2] K. Vintiša, Kompetenču vārdnīca, Creative technologies, 05 (2011)

[3] L. Mitchell, R. Flin, J. Adv. Nursing 63, 15-24 (2008)

[4] L.I. Rabøl, D. Østergaard, T. Mogensen, Quality and Safety in Health Care, 19-27 (2010)

[5] J. McFetrich, Emerg. Med. J. 23, 509-511 (2006)

[6] D.T. Cowan, J. Wilson-Barnett, I.J. Norman, Nurse Educ. Today 27, 452-458 (2007)

[7] R. Meretoja, H. Isoaho, H. Leino-Kilpi, J. Adv. Nursing 47(2), 124-133 (2004)

[8] L. Mitchell, R. Flin, Analysing Behaviour in the Operating Theatre Scrub Practitioners' List of Intra-Operative Non-Technical Skills - SPLINTS (Ashgate, 2009)

[9] M.A. Makary, J.B. Sexton, J.A. Freischlag, et al. J. Am. Coll. Surg. 202(3), 746-52 (2006)

[10] P. Mills, J. Neily, E. Dunn, Teamwork and communication in surgical teams: implications

[11] A. Pike, Current issues in nursing, edited by Dochterman and H. Grace, 6th edn. (St. Louis, MO: Mosby, 2001), pp. 448-452 